\title{
La littérature à l'épreuve de l'occulte
}

\section{Daniel Bornemann}

\section{(2) OpenEdition}

\section{Journals}

Édition électronique

URL : https://journals.openedition.org/rbnu/3225

DOI : $10.4000 /$ rbnu.3225

ISSN : 2679-6104

\section{Éditeur}

Bibliothèque nationale et universitaire de Strasbourg

\section{Édition imprimée}

Date de publication : 1 novembre 2011

Pagination : 76-85

ISSN : 2109-2761

\section{Référence électronique}

Daniel Bornemann, «La littérature à l'épreuve de l'occulte », La Revue de la BNU [En ligne], 4 | 2011, mis en ligne le 01 novembre 2011, consulté le 08 août 2021. URL : http://journals.openedition.org/rbnu/ 3225 ; DOI : https://doi.org/10.4000/rbnu.3225

\section{(c) (i) (2) (-)}

La Revue de la BNU est mise à disposition selon les termes de la Licence Creative Commons Attribution - Pas d'Utilisation Commerciale - Partage dans les Mêmes Conditions 4.0 International. 


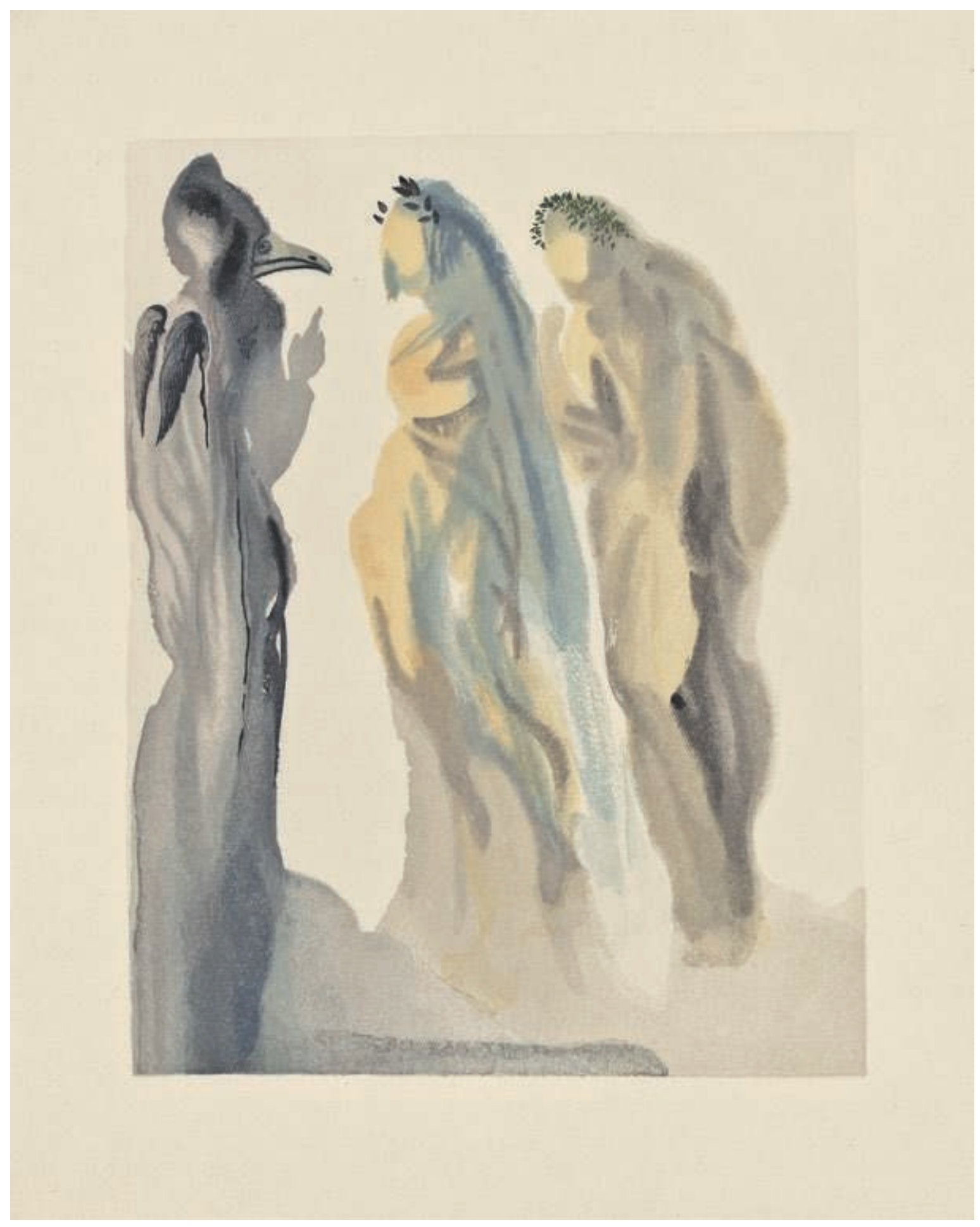

Dante Alighieri. - La Divine Comédie (chant 9 du Paradis).

Illustrations de Salvador Dali. - Paris : Les heures claires, 1963 (Coll. BNU) 


\section{LA LITTÉRATURE À L'ÉPREUVE DE L'OGGULTE}

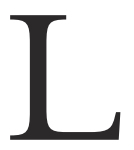

a littérature est le véhicule des mythes qui forment l'imaginaire occidental. Le savoir mythologique occidental trouve ses origines dans les épopées babyloniennes, dans les récits religieux égyptiens et les cosmogonies méditerranéennes. Les livres révélés de la Bible, de la Genèse à l'Apocalypse, ont mis en place ce jeu du dévoilement et de l'occultation qui fait le mystère de la relation entre parole transcendantale et connaissance humaine. Ces dits qui traversent les temps appartiennent au spectre de la littérature, dont le périmètre englobe la philosophie et les corpus religieux. Mais sont-ils littéraires par nature ? Je voudrais tenter d'en discuter.

Ces récits anciens s'enracinent dans ce qu'André LeroiGourhan appelle la " mentalité primitive ", ce qui les exclut de toute origine littéraire. L'idée de chute et de réintégration, autrement dit la plongée dans l'abîme de la mort et la remontée vers la lumière, est un schème plus élaboré qui structure nombre de récits. Dans l'épopée de Gilgamesh et dans l'Enéide, la descente aux enfers est une épreuve type que rencontrent certains héros. Cette exploration, et le récit qui en découle, est le dévoilement d'un aspect du monde auquel le vivant n'a normalement pas accès, et dont le mort ne peut plus rendre compte. La parole de l'auteur, du poète ou de l'anonyme, délivre son message et transgresse l'impossibilité narrative de ce qui est en dehors de l'expérience des vivants. Le discours est donc comparable à la parole du prêtre qui dévoile le secret, qui professe une foi et initie celui qui peut y accéder ; il n'appartient pas à l'activité de l'artiste du langage. Dès lors, le statut de ces textes semble différer de ce que nous nommons littérature. Leur caractère sacré, au sens d'interdit au commun, entre en contradiction avec un schéma de la relation littéraire du lecteur au texte : ces écrits supposent une adhésion au caractère de vérité du dit, qui ne s'accorde plus avec la modalité du contact entre le lecteur et la création d'un poète ou d'un littérateur. La réception de ces textes ne saurait s'effectuer sur le mode de ce que nous appelons le littéraire car il s'agit d'une production révélée. Le lecteur distancié, l'esthète, semble passer à côté de l'essentiel ésotérique ou occulte ; il n'accède donc pas à la totalité du sens, malgré tout son raffinement ou sa culture. Apprécier l'oeuvre pour elle-même, dans sa dimension littéraire, c'est paradoxalement rester à la superficie du texte, aussi profonde soit l'analyse sémantique ou la pénétration intellectuelle. La compréhension de ces écrits semble réclamer une réceptivité " primitive " que l'approche cultivée rend peut-être impossible.

Orphée est à la fois l'archétype du poète absolu et l'origine d'un culte initiatique. On ne conserve rien de ses hymnes, et pourtant la puissance effective de son chant continue de fasciner : il a rythmé la nage des Argonautes, il a couvert l'appel des sirènes, ému les animaux des bois ; les dieux des enfers se sont laissés charmer. Cette poésie touche le monde, le destin, et les change ; c'est cette capacité d'action réelle qui la place au sommet mythique de l'art du poète. La connaissance intime des harmonies naturelles permet 
à Orphée d'ajouter deux cordes à la lyre d'Apollon, ce qui n'est pas peu de chose. Son assassinat, son démembrement par les bacchantes a mis fin à son existence terrestre mais les mystères d'Eleusis, une religion initiatique à laquelle se mêle une part du mythe égyptien d'Osiris sont son oeuvre, nous dit-on. Mais la poésie d'Orphée reste dans l'inconnaissable, car sa voix s'est perdue : ne restent que quelques textes apocryphes, et sa légende, racontée par d'autres poètes. L'aspect qui me semble poser problème est celui d'une création perdue dans le mythe. Cet auteur mythique appartient donc à une civilisation encore orale. Le message orphique était ce qui se disait ou se savait de ses hymnes, et ce qui se transmettait dans le secret du temple d'Eleusis. L'adepte emportait ce trésor dans son coeur, sans jamais le révéler. Il nous reste donc l'orphisme, dont on connaît à peine quelques linéaments, et un prince des poètes de l'Antiquité, fils d'une muse, dont l'oeuvre reste à jamais inconnue.

La Divine comédie (1321) pose elle aussi quelques difficultés. Dante met en poème le songe du milieu de sa vie, sous la forme d'un voyage à travers les trois règnes de l'Au-delà. Littérature et religion trouvent, dans les cent chants en " terza rima ", la géométrie d'une cathédrale chantant la vie, la mort et la résurrection. Lecture littéraire, lecture chrétienne, dans la distanciation esthétique ou dans la foi, ou encore lecture historique, dans l'érudition, et enfin lecture ésotérique, cette oeuvre prête à une pluralité de perceptions. Pour certains connaisseurs, qui comme René Guénon ont de bonnes raisons pour l'affirmer, la dimension ésotérique occupe le sommet hiérarchique de l'oeuvre. La force créatrice mise en action par l'auteur en fait certes un chef-d'oeuvre incontesté de la poésie mondiale, mais si l'essentiel est ailleurs que dans la poésie, au-delà de l'art du langage lui-même, son statut ne dévie-t-il pas du littéraire vers un autre domaine ? Si la Comédie est un manifeste, reste-t-elle une construction du langage ou devient-elle l'expression cryptée d'une sagesse, d'un savoir caché, de quelque chose qui dépasse la littérature ? Les signifi- cations extérieures fournissent un mode d'expression pour des vérités supérieures et ne sont qu'un symbole, un reflet de l'intelligible, qui reste donc non-lisible. La perfection lisible de l'oeuvre littéraire ne serait alors qu'un reflet de l'autre perfection, indicible car secrète, qui serait celle de la tradition ésotérique ellemême : celle des Templiers, des rose-croix, de la quête du Graal et de la maçonnerie. Comme le Roman de la Rose, la Divine comédie trouverait son sens au-delà du littéraire et échapperait alors aux critères de la littérature. L'approche littéraire serait inopérante, ou seulement accessoire, inessentielle. René Guénon dit que ce qui est exprimé dans la Comédie n'est pas de la littérature mais de la vérité, tout simplement. La beauté des vers, la perfection des rimes, des rythmes ou du traitement des thèmes ne seraient qu'un effet de surface qui détourne l'attention de l'essentiel de la signification.

A la Renaissance, le néoplatonisme redécouvre et traduit des textes fondateurs issus de la tradition hellénistique et grecque, notamment le corpus hermétique et les oeuvres de Platon (vers 1463). Ce mouvement ébranle les certitudes aristotéliciennes. La vie littéraire prend à ce moment un tournant qui l'amène à entrer en dissidence par rapport à la civilisation chrétienne. L'emploi des thèmes mythologiques classiques s'intensifie et devient dominant. Le Songe de Poliphile (1467), écrit par Francesco Colonna, calque sa structure d'ensemble sur la Divine comédie mais fait évoluer le protagoniste dans un monde de réminiscences antiques. L'ouvrage est ésotérique par bien des aspects, notamment par la langue particulière dont il est constitué, mais aussi et surtout par le goût du mystère, de l'allégorie et des jeux de symboles souvent abscons. Cette volonté manifeste de l'auteur de dérouter les interprètes ou de les soumettre à un jeu de pistes par trop compliqué pose problème dans ce livre. L'ouvrage est resté célèbre surtout pour la qualité de son illustration, de sa typographie et de sa beauté plastique, issue des presses d'Alde Manuce (1499), et passe à juste titre pour le plus bel in- 


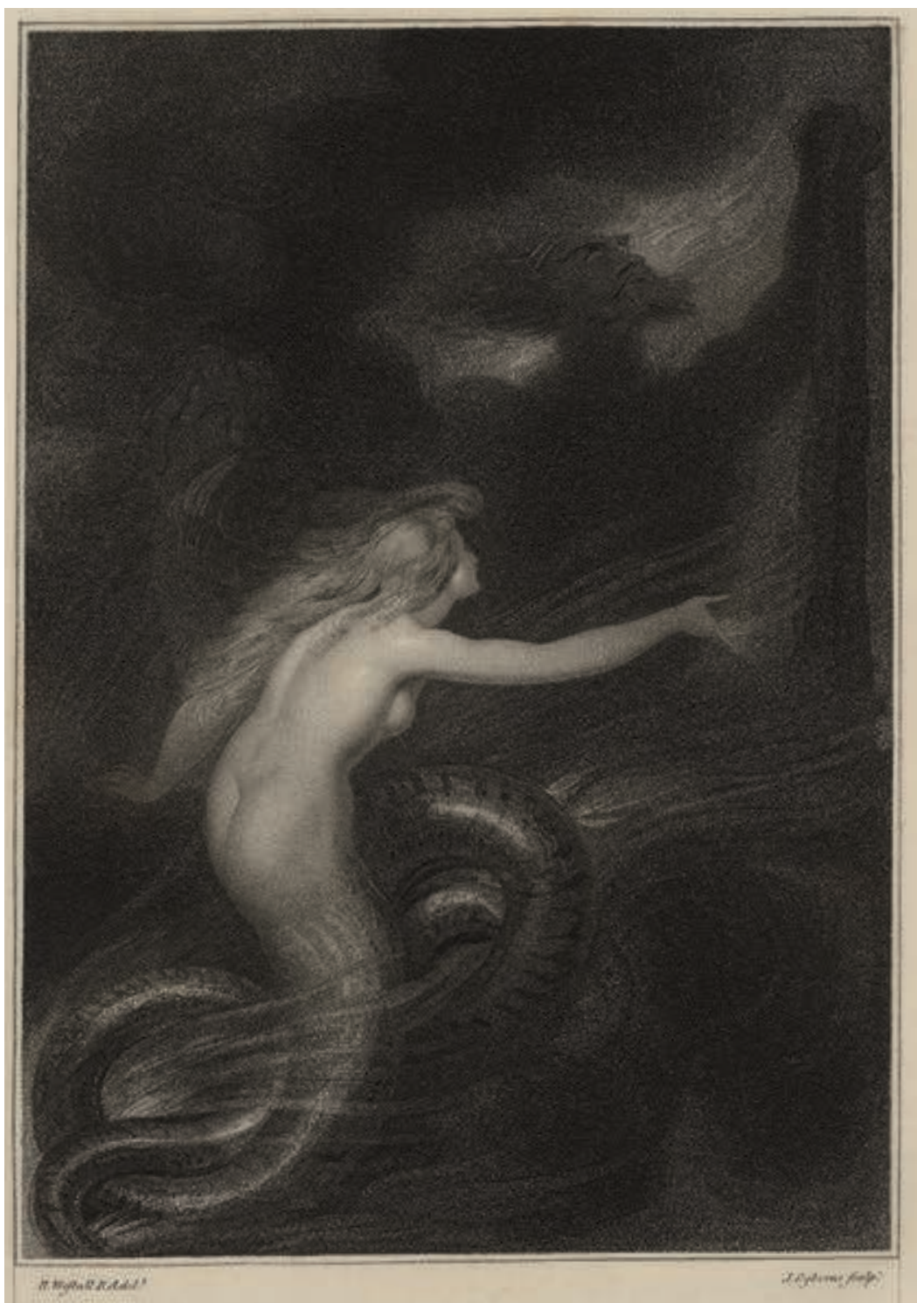

John Milton. - The poetical Works : Paradise Lost.

With a life of the author by William Hayley. -

Londres : W. Bilmer, 1795 (Coll. BNU) 


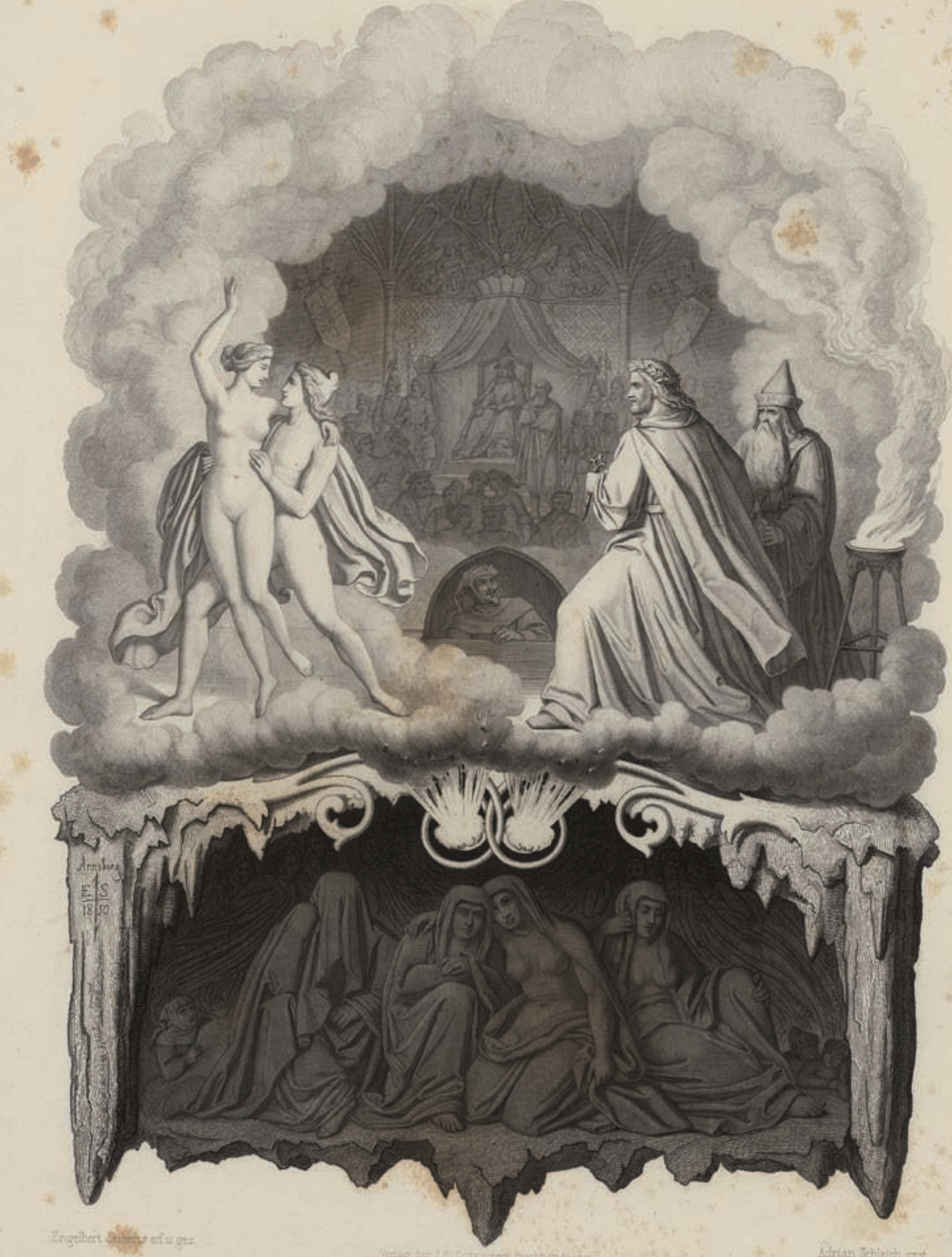


cunable jamais imprimé. L'approche littéraire trouvet-elle son compte dans cette oeuvre ? Elle ne parvient pas à rendre compte de l'ouvrage, car ce sont d'autres dimensions qui lui confèrent son aura, cet ensemble de significations qui est son sens. L'oeuvre des artistes du livre, graveurs et typographes, appartient plus à la " raison graphique " qu'au domaine de la littérature. L'approche littéraire de cette oeuvre, à la fois ouverte et fermée, reste problématique.

$\mathrm{Au}$ cours de la Renaissance, la philosophie s'est scindée de la métaphysique chrétienne. La divinité ellemême change de nature aux yeux des philosophes et des poètes de l'avant-garde. Dieu devient

" flux et reflux ", " chaleur ", ou

encore " soleil ", et cette nouvelle

donne accorde plus de liberté

aux poètes pour traiter chacun

à sa manière les questions

divines. John Milton se

saisit du sujet biblique de

la Chute, et se donne liber-

té de le réécrire dans son

Paradis perdu (1667). Milton

est un protestant porté

vers une sorte de maté-

rialisme issu de sa pensée

religieuse elle-même. Il n'y a

pas de place pour le mystère

dans sa théologie. Il est en fait

totalement libéré de tout système

religieux établi, et il peut se saisir de

l'histoire de la chute d'Adam et d'Eve, de

ce passage de la Genèse, et mettre son imagination en

oeuvre dans un acte poétique de grande ampleur pour

en déployer tous les détails, et en inventer tous les

dialogues. Il fait parler Satan, Dieu, les anges... mais

il entreprend également de retracer l'histoire des doctrines mystiques de l'Orient méditerranéen et donc de l'Occident. Cette poésie philosophique est basée sur la tradition chrétienne mais celle-ci est vue avec des yeux différents, ceux d'un aveugle, mais qui ont leur propre lucidité. L'auteur a lu certains livres de la kabbale et retenu l'idée du retrait de Dieu, de son absence partielle des destinées du monde. Son oeuvre est une réécriture du mythe fondateur judéo-chrétien, dont le caractère religieux et sacré a été écarté, au profit d'une sacralité nouvelle : celle d'un mythe poétique personnalisé. La langue qui imite la diction des Anciens et s'écarte de la syntaxe de l'anglais de son époque, donne également à l'oeuvre une dimension ésotérique. Certains passages, certains vers, présentent une difficulté que Chateaubriand, son traducteur, appelle des " obscurités invincibles ". Il y a une volonté de non dévoilement du sens, de dissimulation. L'oeuvre à la fois dévoile et obscurcit, permet au lecteur, comme elle l'a permis au poète, de toucher au sacré, mais en même temps rompt ce rapport au sacré, au révélé. Faire oeuvre de littérature à partir du mythe biblique de la chute revient donc à désacraliser, à déconsacrer la Genèse. Le rapport de l'homme au divin, d'Adam à Satan, du lecteur à la vérité révélée, se trouve dévoyé. La révolte d'un auteur face à la révélation et au sacré rend problématique et personnelle la relation au transcendant. La littérature ici nous sépare du contenu ésotérique de la Bible, livre révélé, dans ce qu'il comporte d'initiatique.

La thématique du lien de l'humain au divin, au surnaturel, de la relation entre le vivant et l'éternel, avec les esprits et les puissances de la magie, nourrit abondamment la création littéraire, depuis ses origines. D'autres thèmes comme celui du double ou du " mort-vivant" ont produit d'innombrables oeuvres qui forment le genre fantastique. Le mystère, l'action de l'inexplicable, sont de puissants moteurs de l'imaginaire et suscitent les récits à l'infini. Cependant, lorsque ces mystères sont utilisés en littérature comme ingrédients, comme ressorts techniques dans la narration, lorsqu'une instrumentalisation transparaît, il se crée une distanciation qui met à mal la dimension occulte. Un initié n'accordera pas autre chose qu'un regard amusé et un sourire condescendant aux créations de la littérature fantastique. S'il y trouve quelque élément qui entre en résonance avec ses convictions, il y portera son attention, au détriment de l'attention à l'oeuvre elle-même, à sa construction et à la qualité de son expression. L'ésotériste ne considérera l'artefact littéraire que comme une fantaisie visant le délassement, l'amusement, la délectation du lecteur. Sa propre recherche, ses intuitions, ses convictions ou ses expérimentations le 


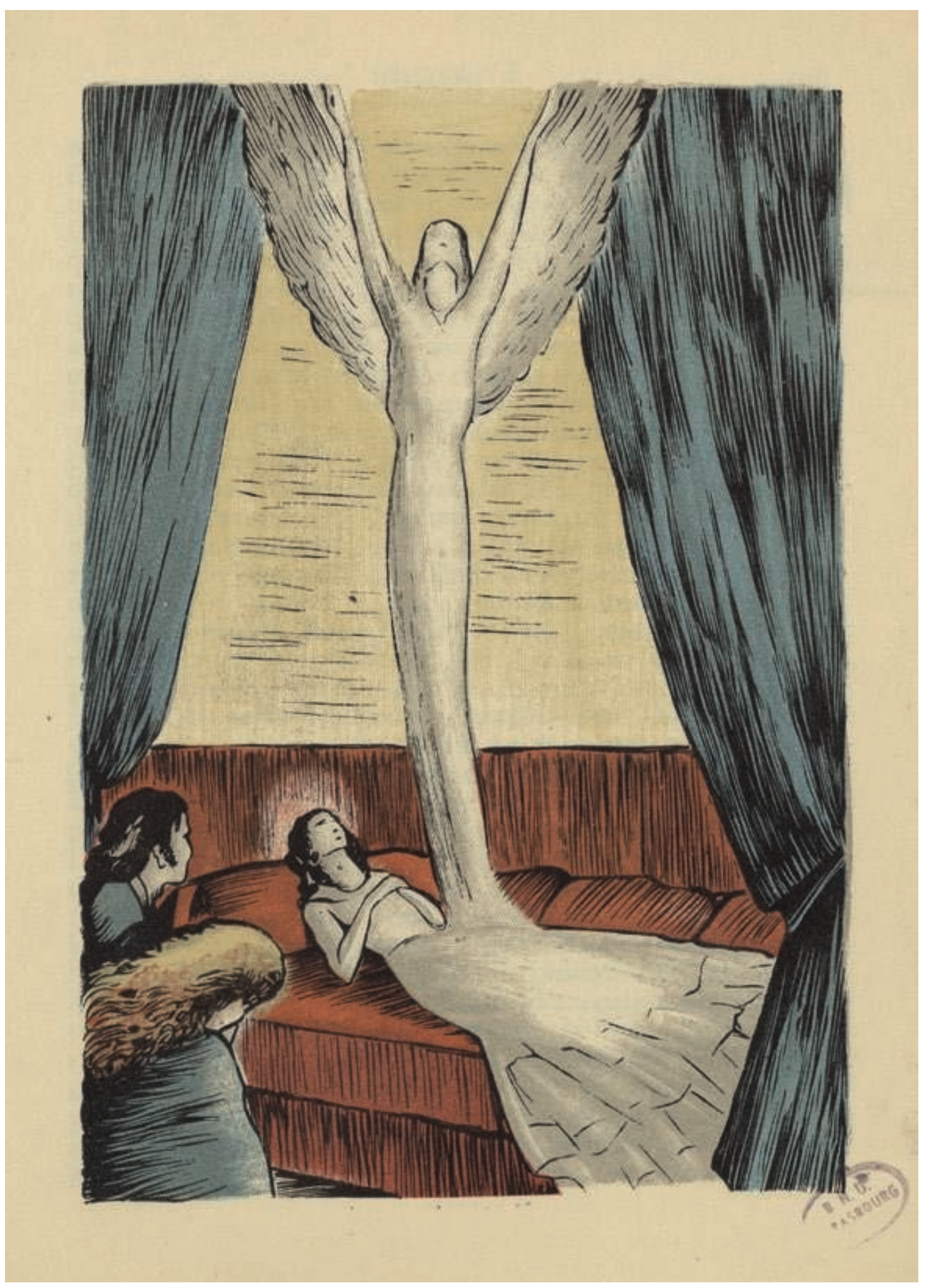

Honoré de Balzac. - Séraphita : extrait du livre mystique.

Dessins de Thomas Sarti, gravés sur bois

en couleurs par Etienne K. Heywand. -

Paris : Albert Guillot, 1953 (Coll. BNU) 


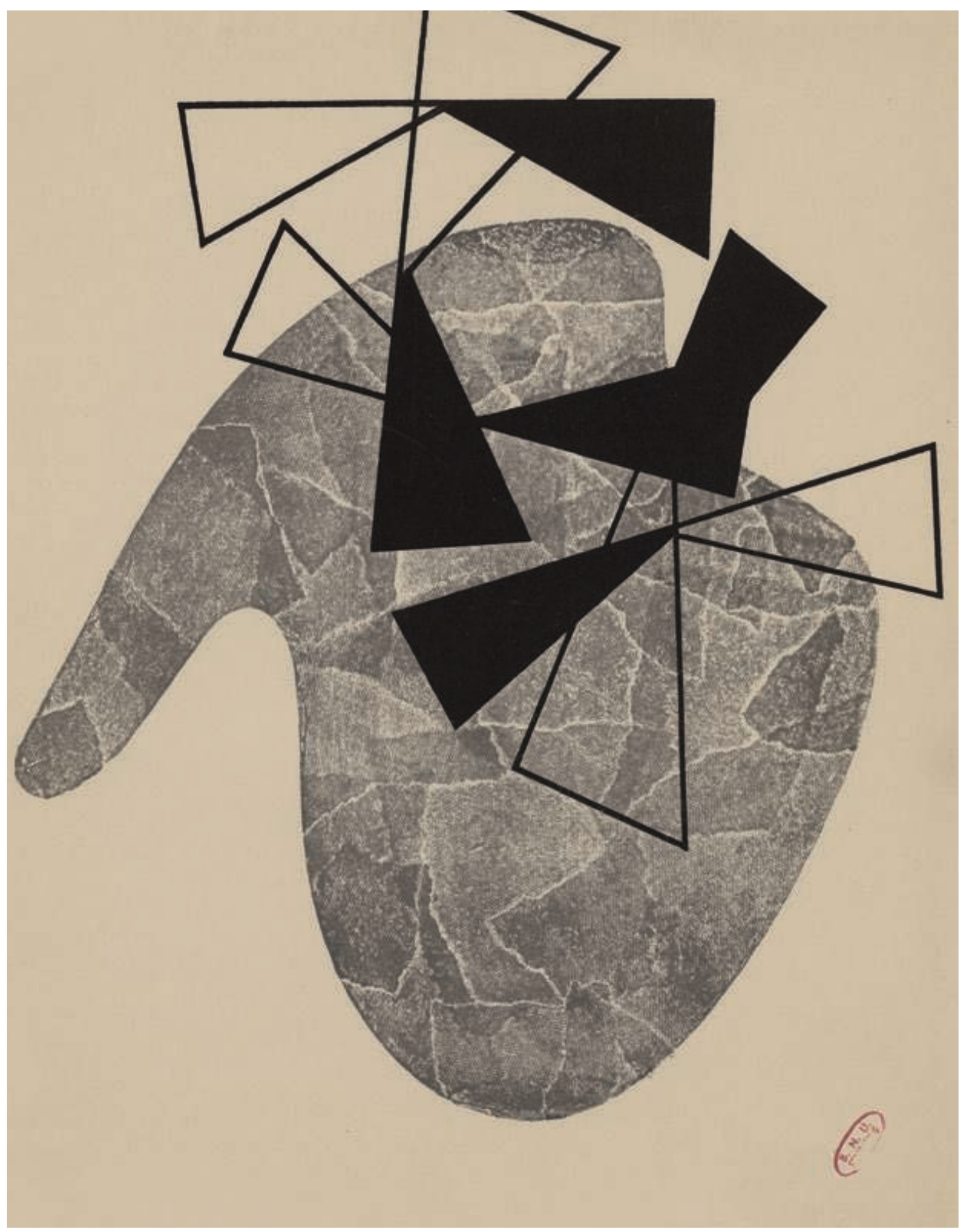

Jean Arp. - Die Engelschrift. - Tübingen : H. Laupp junior, 1952

Typographie selon les indications de Jan Tschichold

Exemplaire numéroté 52 (Coll. BNU) 
détourneront de ces constructions esthétiques pour le renvoyer vers ses méditations. August Strindberg traite de ce rapport de l'occultiste au roman fantastique dans Inferno, où le héros lecteur du Séraphita de Balzac se tourne vers l'oeuvre de Swedenborg, autrement plus éclairant.

Le demi-scepticisme des temps modernes s'accommode fort bien des poussées fantastiques qu'enregistre l'histoire de la civilisation. Les Faust de Goethe (17731832) utilisent un vieux récit pour exalter les infinis pouvoirs de l'imagination et poussent les acquis de la tradition occultiste vers les extrémités de la fantaisie, jusqu'à les dépasser, en créant soudain un fragment de mythologie nouvelle, personnelle : le passage par les " mères " qui, tapies dans la caverne des esprits, restent les mystérieuses créatrices et assembleuses des êtres. Goethe s'appuie sur tous les éléments connus de l'occultisme pour mettre au jour un mythe personnel, qui a même plus d'importance à ses propres yeux que tout le reste de son oeuvre, laisse-t-il entendre à Eckermann à la date du 10 janvier 1830. Le Goethe du Second Faust, donc celui des Conversations, a bel et bien foi en ces " mères", et aussi en un au-delà de la vie terrestre, chrétien ou non, et cette foi l'écarte de ce monde fantastique, auquel il a pourtant travaillé durant toute sa vie. Sa foi, peu définie mais cependant affirmée, le sépare en fin de compte de son chef-d'oeuvre théâtral, qui dès lors perd de son aura pour ne redevenir qu'une réussite littéraire parmi d'autres du grand auteur.

Ces problèmes d'adhésion ou non aux dimensions occultes ou ésotériques, qui creusent à mon sens un fossé entre recherche mystique et création littéraire, trouvent en Victor Hugo un autre exemple, et pour ainsi dire une solution. On sait que la mort de sa fille Léopoldine en 1843 a divisé en deux parties la vie du poète. Le mesmérisme et certains aspects de l'occultisme lui étaient connus de longue date, mais le désespoir de cette perte chère le poussa à tout tenter pour rétablir le contact avec elle à partir du moment où, en 1853 , le spiritisme fit son entrée en Europe. Grâce à plusieurs "illuminés de son temps ", il crut communiquer avec la défunte; il prit goût à ces expériences spirites et put converser avec d'autres auteurs comme Dante ou Shakespeare. A partir de ce moment, ses compositions s'imprègnent de spiritisme, de mystique, d'occultisme et de caractère visionnaire. Les contemplations, La légende des siècles, Dieu et La fin de Satan, toute la production de la seconde moitié de sa vie est imprégnée de métaphysique, de ce contact avec le surnaturel, et confirment l'auteur en tant que mage. Libéré des contraintes de la religion chrétienne, qui interdit la prise de contact avec les morts, il écrit sans limitations sa vision d'envergure, exprimée par analogies et symboles. Les adeptes de l'occultisme, Eliphas Lévi et Saint-Yves d'Alveydre, savouraient ces poèmes et virent en lui " un grand magicien ".

Avec Milton, Goethe et Hugo, l'occultisme a trouvé trois interprètes, sans pourtant que le lien à ces systèmes soit plus profond qu'une parenté plus ou moins prononcée. Les sciences occultes, ésotériques, sont multiples, même si la tradition est censée être une. Ces créations littéraires sont également personnelles, ce qui pose là encore problème : celui de la crédibilité, nécessaire à l'adepte convaincu. La démarche des poètes s'écarte de celle des chercheurs d'absolu en cela qu'elle est recherche de l'expression parfaite d'une vision intérieure personnelle, et non de l'exposé d'un savoir acquis par l'initiation. La métaphysique des poètes est une sorte de refuge philosophique ourlé de bonheur d'expression et s'adressant à la sensibilité, alors que les systèmes de l'occultisme sont une recherche de la vérité cachée. Entre ces deux pôles de l'activité intellectuelle, il reste une frontière. C'est ce que Denis Saurat relève dans La littérature et l'occultisme. La recherche de l'expression heureuse s'adresse à la sensualité littéraire, en quête de qualité de langage et d'image. Les mystiques, quant à eux, brident toujours leur sensualité, car c'est dans l'ascèse que l'élévation vers l'inaccessible est selon eux possible. On n'accède pas par les sens à la vision intérieure mais par l'abstraction.

René Guénon quant à lui explique qu'il est des "vérités " qui ne s'écrivent pas. Il bride lui-même son expression lorsqu'il les effleure, pour dénoncer l'Erreur spirite, pour démasquer la pseudo-religion qu'est le " théosophisme ", et lorsqu'il lui faudrait lever un coin du voile d'Isis, il craint les " applications pratiques que certains ne manqueraient pas de vouloir en tirer ". Cette crainte vient donc directement de la croyance à l'effectivité des savoirs surnaturels. Le même voit dans la littérature fantastique une " propagande indirecte " qui est " peut-être la plus malfaisante de toutes parce qu'elle [...] assure la plus grande diffusion aux théories en question ". Selon les spécialistes, ces choses sont trop graves pour en faire de la littérature.

Les données secrètes de l'occultisme s'accommodent mal de l'expression écrite. Les alchimistes créaient des 
pentacles pour dévoiler leurs arcanes, en évitant l'écriture suivie. Les livres d'emblèmes évitaient, contournaient l'écriture pour encoder autrement le savoir ésotérique. Ce domaine du savoir ne relève pas vraiment de la civilisation écrite mais d'une part de notre culture qui est restée partiellement orale. Il relève du domaine des secrets et non de ce qui se rend public. La part publiée n'est pas essentielle, bien au contraire, et elle est soit mensongère, soit tellement parcellaire qu'elle ne renseigne pas sur le coeur du sujet. Les grands succès de librairie, ceux de Paulo Coelho par exemple, maintiennent dans le public une disposition et une curiosité sympathisante envers ces savoirs réservés. Mais les occultistes s'en méfient.

$\mathrm{Au}$ début du vingtième siècle, l'insatisfaction face au discours réaliste comme face au symbolisme est à l'origine d'une réaction qui consiste à aller chercher ailleurs que dans la pensée construite l'inspiration poétique et artistique. Cette mise en cause de la construction consciente du discours a permis à certains auteurs de se libérer de cette dichotomie réalitél fiction. Jean Arp a dans ses poèmes et dans ses gravures mis en scène des voix, celles des anges, celles des choses, et représenté dans une quasi-abstraction la présence d'esprits dans l'indéterminé de l'espace blanc. André Breton a utilisé l'écriture et le dessin spirites, dans Nadja et dans bien d'autres créations. Les surréalistes ont volontiers exploité dans leurs essais l'imagerie issue de la tradition ésotérique en tant que stimulants intellectuels capables de bousculer le rationnel. En effet, l'occultisme, le spiritisme ne sont-ils pas voyages dans la sur-réalité, dans une réalité supérieure?

Cette réussite, due à leur recherche d'indétermination, d'abstraction, et à l'usage d'une alchimie littéraire, a permis aux traditions ésotérique, hermétique, occultiste, une nouvelle période de vitalité, cette fois-ci en dehors de l'adhésion mentale réfléchie, qui pose les problèmes soulevés jusqu'ici. Y aura-t-il encore d'autres semblables feux d'artifice dans la civilisation ? Le moyen en sera certainement une nouvelle manière d'enjamber l'abîme qui sépare poésie et vérité.

En somme, la difficulté du rapport entre littérature et occultisme ressemble à l'antagonisme entre l'humide et le gras, qui permet sur la pierre lithographique la composition d'une image faite de vides et de surfaces encrées. La littérature et l'occultisme semblent se fuir et se poursuivre, s'écarter et adhérer à leurs contours extérieurs, réciproquement. Le noir, le blanc et toutes les couleurs forment l'image même, celle d'un imaginaire, ou comme le disait Henry Corbin, un " imaginal " qui dessine un monde intermédiaire entre l'intelligible et le perceptible. De grands poètes et d'innombrables écrivains ont tenté cette écriture, jeu d'encres et de fluides sur la pierre philosophale, proposant chacun sa manière de résoudre ou d'escamoter ce difficile rapport entre rêve et réalité.

\section{Daniel Bornemann}

